Case Report

\title{
Second Trimester Heterotopic Triplet Pregnancy with Intrauterine Twin Pregnancy and Ruptured Interstitial Pregnancy: A Maternal Near-Miss Case Report
}

\author{
Charles Nkurunziza $\mathbb{D}^{1}{ }^{1}$ Theogene Rurangwa, ${ }^{2}$ Vincent Ngendahimana, ${ }^{2}$ \\ and Urania Magriples ${ }^{3}$ \\ ${ }^{1}$ University of Rwanda, Department of Obstetrics and Gynecology, Rwanda \\ ${ }^{2}$ Rwanda Military Hospital, Department of Obstetrics and Gynecology, Rwanda \\ ${ }^{3}$ Yale University School of Medicine, Division of Maternal Fetal Medicine, USA \\ Correspondence should be addressed to Charles Nkurunziza; nkurunziza86@gmail.com
}

Received 26 November 2019; Accepted 28 February 2020; Published 11 March 2020

Academic Editor: Giovanni Monni

Copyright (c) 2020 Charles Nkurunziza et al. This is an open access article distributed under the Creative Commons Attribution License, which permits unrestricted use, distribution, and reproduction in any medium, provided the original work is properly cited.

Heterotopic pregnancy is defined as the occurrence of simultaneous intrauterine and extrauterine pregnancies. It is a rare, potentially life-threatening condition and infrequent in natural conceptions. Here, we report a case of spontaneous heterotopic triplet pregnancy with ruptured cornual ectopic pregnancy and simultaneous twin intrauterine pregnancies at 18 weeks of gestation. The event led to miscarriage of all fetuses from both the ectopic and the intrauterine twin pregnancies.

\section{Introduction}

Heterotopic pregnancy is defined as the occurrence of simultaneous intrauterine and extrauterine pregnancy [1]. It is a rare and potentially life-threatening condition which is infrequent in natural conception cycles with the incidence of 1 in 30,000 pregnancies $[1,2]$. Its occurrence rises to $1: 100$ to $1: 500$ with the use of assisted reproductive technologies $[1,2]$. We report a case of spontaneous heterotopic triplet pregnancy with an intrauterine twin pregnancy and ruptured cornual pregnancy diagnosed at 18 weeks of gestation. The rupture of the cornual pregnancy resulted in miscarriage of all fetuses from both the ectopic and the intrauterine twin pregnancies.

\section{Case Presentation}

A 34-year-old G6P2122 at 18 weeks of gestation with a history of 2 prior cesarean deliveries was transferred to the Rwanda Military Hospital (RMH) in Kigali, Rwanda, in hypovolemic shock. She had one prior antenatal visit but no ultrasound was done. Her history was also remarkable for pregnancy on IUD contraception. She was seen at a health center with a history of sudden onset severe abdominal pain followed by general body weakness and was referred to a district hospital where ultrasound showed miscarriage of an intrauterine twin pregnancy at 18 weeks of gestation and an additional intra-abdominal fetus dead as well. They suspected uterine rupture and transferred the patient to $\mathrm{RMH}$ after initiating IV fluids and transfusion. Travel time was approximately 3 hours, and she reached RMH 12 hours after the onset of symptoms in hypovolemic shock with tachypnea and tachycardia of $144 \mathrm{bpm}$. She was pale with a distended abdomen and rebound tenderness. Point of care ultrasound showed an intrauterine twin pregnancy with no cardiac activity for both fetuses. A third fetus was seen outside the uterus without cardiac activity and there was free fluid in the abdomen. Results of the full blood count (FBC) at admission came with $\mathrm{Hb}$ of $11.6 \mathrm{~g} / \mathrm{dL}, \mathrm{WBC}$ of $28.31 \times 10^{3} / \mu \mathrm{L}$, and Plt of $280 \times 10^{3} / \mu \mathrm{L}$. The patient was immediately taken to the operating room for explorative laparotomy, and consent for possible hysterectomy was signed. Intraoperatively, 


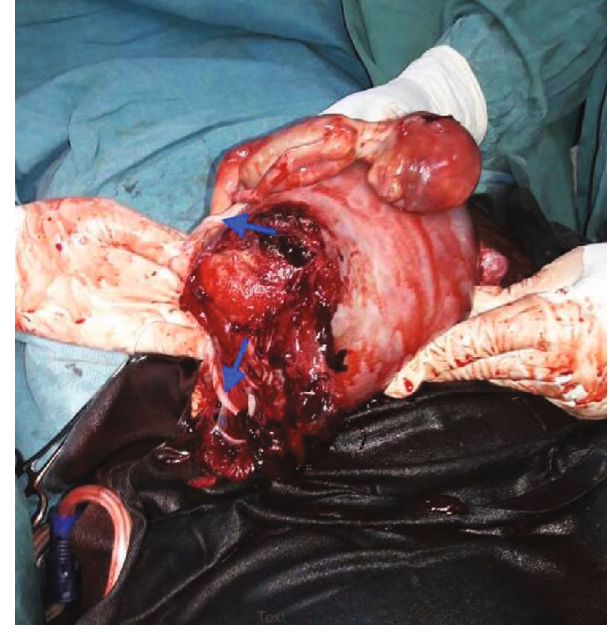

Figure 1: Fetus from the ruptured right interstitial pregnancy alongside the gravid uterus after its exteriorization. The placenta and umbilical cord are still attached to the ruptured site as shown by the blue arrows.

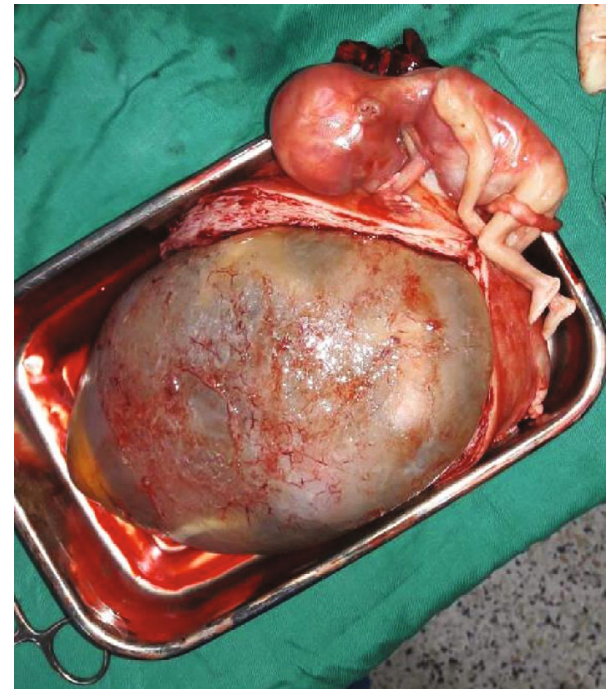

FIGURE 2: Fetus from ruptured interstitial pregnancy with an open uterus and intrauterine pregnancy membranes still intact.

approximately $4 \mathrm{~L}$ of blood and clots was aspirated. A right side interstitial ruptured pregnancy with a dead male fetus in the abdomen with its placenta still attached to the ruptured site was found (Figure 1). The uterus was not reparable because of the extent of the cornual rupture, and a subtotal hysterectomy was performed. Postoperatively, the uterus was opened and a monochorionic-diamniotic twin intrauterine pregnancy was noted (Figures 2 and 3 ). The patient received blood products intraoperatively and postoperatively. She fully recovered from anesthesia and was discharged from the hospital on her $4^{\text {th }}$ day post operation.

\section{Discussion}

Heterotopic pregnancy is rare in spontaneous natural pregnancy with the incidence of 1 in 30,000 pregnancies, and het-

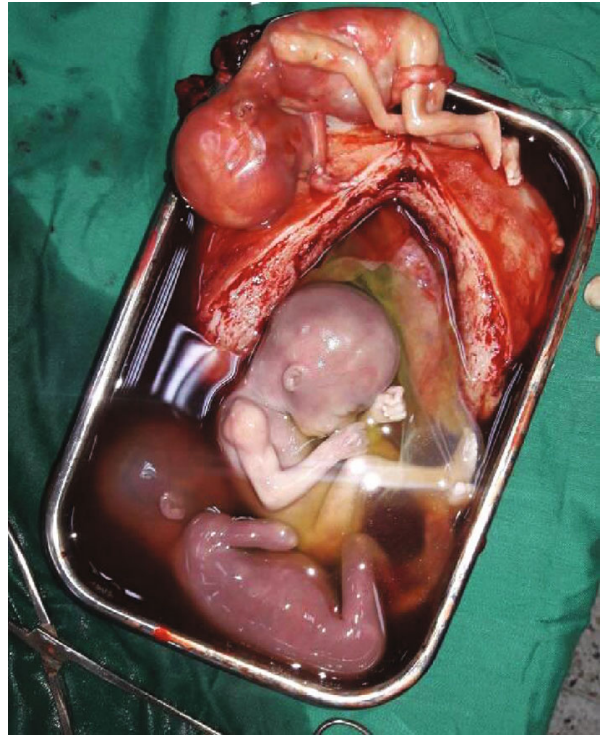

FIgURE 3: Intrauterine twin pregnancy after rupturing the membranes, with the fetus from the ruptured interstitial pregnancy.

erotopic triplet pregnancy with ectopic pregnancy coexisting with twin intrauterine pregnancy is rarer $[1,3]$. Risk factors for heterotopic pregnancy are similar to risk factors for ectopic pregnancy and include previous ectopic pregnancy, pelvic inflammatory disease, infertility, in vitro fertilization, previous tubal reconstructive surgery or pelvic surgery, failed contraceptive methods like intrauterine devices and tubal sterilization, and smoking [4-7]. For our case, the identified risk factor was a failed intrauterine device (IUD) as she had conceived on IUD contraception. A high index of suspicion and transvaginal ultrasound are important in timely diagnosis of heterotopic pregnancy especially in spontaneous pregnancies [1]. Early transvaginal ultrasound performed by an experienced sonographer has both high sensitivity and specificity in diagnosing heterotopic pregnancy [8]. The timing of the diagnosis of heterotopic pregnancy is variable from 5 to 34 weeks with the majority being diagnosed between 5 and 8 weeks of gestation [2]. When the diagnosis is made before rupture of the ectopic pregnancy, the prognosis for the intrauterine pregnancy is good [1]. However, timely diagnosis is only possible where the prenatal ultrasound is available, and women seek for antenatal consultation early in pregnancy. In our case, the diagnosis was made late as she was in hypovolemic shock on presentation. The diagnosis of heterotopic pregnancy is not easy but adequate training of primary and secondary care doctors and/or sonographers in early pregnancy ultrasound can help in achieving timely detection of heterotopic or simply ectopic pregnancies [8].

\section{Conclusion}

A high index of suspicion is essential to diagnosis of heterotopic pregnancy especially in spontaneous pregnancies, and clinicians should be aware that confirming an intrauterine pregnancy does not exclude the risk of ectopic pregnancy. Clinicians performing antenatal ultrasound 
scans should thoroughly scan the pelvis to rule out an extrauterine pregnancy despite having confirmed the intrauterine pregnancy.

Educating women for early first antenatal consultation to confirm the location of pregnancy and improving access to ultrasound in antenatal clinics is the key to saving lives of women from heterotopic pregnancies or ectopic pregnancies burden.

\section{Abbreviations}

BP: Blood pressure

FBC: Full blood count

EGA: Estimated gestational age

g/dL: Grams per deciliter

GCS: Glasgow coma scale

$\mathrm{Hb}$ : Hemoglobin

HR: Heart rate

IUD: Intrauterine device

IV: Intravenous

Plt: $\quad$ Platelets

RMH: Rwanda Military Hospital

RR: Respiratory rate

SPO2: Oxygen saturation

$\mu \mathrm{L}: \quad$ Microliter

US: Ultrasound

WBC: White blood cells.

\section{Consent}

Patient's written consent for publication was obtained for publication of this case.

\section{Conflicts of Interest}

No competing interests declared by the authors with regard to research, authorship, and publication of this article.

\section{Authors' Contributions}

$\mathrm{CN}, \mathrm{TR}$, and VN participated in the management of this case and contributed to gathering clinical information and the pictures used. $\mathrm{CN}$ and UM wrote the manuscript report. All authors read and approved the final manuscript.

\section{References}

[1] S. Rengaraj, S. Vinayagam, and S. Soukath, "Case report spontaneous heterotopic triplet pregnancy : rare case report," International Journal of Reproduction, Contraception, Obstetrics and Gynecology, vol. 5, no. 12, pp. 4496-4498, 2016.

[2] L. Arsala and D. Danso, "Spontaneous heterotopic triplet pregnancy with tubal Rupture," Journal of Investigative Medicine High Impact Case Reports, vol. 2, no. 2, pp. 1-4, 2014.

[3] M. Gosavi, "Spontaneous heterotopic triplet pregnancy with tubal ruptured ectopic gestation," Juniper Online Journal of Case Studies, vol. 5, no. 1, pp. 1-4, 2017.

[4] M. Heather, B. Hanadi, B. Trevor, and T. Togas, "Diagnosis and treatment of ectopic pregnancy," Canadian Medical Association Journal, vol. 173, no. 8, pp. 905-912, 2005.
[5] J. Bouyer, J. Coste, T. Shojaei et al., "Risk factors for ectopic pregnancy: a comprehensive analysis based on a large case-control, population-based study in France," American Journal of Epidemiology, vol. 157, no. 3, pp. 185-194, 2003.

[6] J. L. V. Shaw, G. S. Wills, K. F. Lee et al., "Chlamydia trachomatis Infection Increases Fallopian Tube PROKR2 via TLR2 and NF $\kappa \mathrm{B}$ Activation Resulting in a Microenvironment Predisposed to Ectopic Pregnancy," The American Journal of Pathology, vol. 178, no. 1, pp. 253-260, 2011.

[7] C. Li, W. H. Zhao, Q. Zhu et al., "Risk factors for ectopic pregnancy: a multi-center case-control study," BMC Pregnancy and Childbirth, vol. 15, no. 1, pp. 1-9, 2015.

[8] X. H. Li, Y. Ouyang, and G. X. Lu, "Value of transvaginal sonography in diagnosing heterotopic pregnancy after in-vitro fertilization with embryo transfer," Ultrasound in Obstetrics \& Gynecology, vol. 41, no. 5, pp. 563-569, 2013. 